\title{
Urticária Crônica Espontânea e Síndrome de Sjögren: relato de caso
}

\author{
Chronic Spontaneous Urticaria and Sjögren's Syndrome: case report \\ Urticaria Crónica Espontánea y Síndrome de Sjögren: reporte de caso
}

Recebido: 29/11/2021 | Revisado: 04/12/2021 | Aceito: 10/12/2021 | Publicado: 18/12/2021

\author{
Brenda da Silveira Santos \\ ORCID: https://orcid.org/0000-0001-5294-1986 \\ Faculdade Morgana Potrich, Brasil \\ E-mail: brenda.s.santos@aluno.famp.edu.br \\ Gustavo Almeida Linhares \\ ORCID: https://orcid.org/0000-0002-6997-0385 \\ Faculdade Morgana Potrich, Brasil \\ E-mail: gustavo.a.linhares@aluno.famp.edu.br \\ Severino Correia do Prado Neto \\ ORCID: https://orcid.org/0000-0002-3998-1251 \\ Faculdade Morgana Potrich, Brasil \\ E-mail: severinoneto@fampfaculdade.com.br
}

\begin{abstract}
Resumo
Introdução: A Urticária Crônica Espontânea (UCE) se caracteriza por pápulas transitórias, angioedema ou ambos por mais de seis semanas. A UCE pode estar associada à Síndrome de Sjögren (SS). Objetivo: descrever um caso clínico de uma paciente com diagnóstico de Urticária Crônica Autoimune, sem melhora com tratamento padrão. Metodologia: Este estudo de caso foi realizado qualitativamente, descritiva, observacional e retrospectiva, por coleta direta de dados da paciente por meio do acesso aos prontuários e exames. Descrição do caso: paciente do sexo feminino, 34 anos, encaminhada para avaliação reumatológica com quadro de UCE. Na pesquisa de causas secundárias para UCE, apresentou exames de investigação com alterações laboratoriais. Além disso, queixou-se também de sintomas secos. Realizado os exames, concluiu se tratar de um caso de UCE associada à SS. Após tratamento da doença reumática de base, apresentou melhora das lesões urticariformes. Discussão: No caso relatado as lesões foram refratárias mesmo após introdução do tratamento com anti-histamínico. Algumas evidências científicas sugerem maiores taxas de refratariedade ao tratamento padrão da UCE naqueles pacientes que apresentam eventos imunomediados. Além do quadro sugestivo de UCE, a paciente manifestou sintomas secos. Normalmente, as manifestações clínicas não ocorrem de forma simultânea, o que não foi visualizado na paciente já que a mesma apresentou quadros concomitantes. Conclusão: O caso retrata a ocorrência típica de UCE associada à SS e ressalta a importância da realização de uma boa anamnese e exame físico. Além disso, chama a atenção para a associação entre a UCE e outras doenças imunomediadas.
\end{abstract}

Palavras-chave: Urticária crônica espontânea; Síndrome de Sjögren; Autoimune.

\begin{abstract}
Introduction: Chronic Spontaneous Urticaria (CHU) is characterized by transient papules, angioedema, or both for more than six weeks. CHU may be associated with Sjögren's Syndrome (SS). Purpose: This was to describe a clinical case of a patient diagnosed with Chronic Autoimmune Urticaria, with no improvement with standard treatment. Methodology: This case study was conducted in a qualitative, descriptive, observational and retrospective way, by direct patient data collection through access to medical records and exams. Case description: Female patient, 34 years old, referred for rheumatologic evaluation with UCE. In the search for secondary causes for CHU, she presented investigational tests with laboratory abnormalities. In addition, she also complained of dry symptoms. The exams concluded that this was a case of CHU associated with SS. After treatment of the underlying rheumatic disease, he presented improvement of the urticarial lesions. Discussion: In the reported case the lesions were refractory even after introduction of antihistamine treatment. Some scientific evidence suggests higher refractory rates to the standard treatment of UCE in those patients who present with immune-mediated events. In addition to the picture suggestive of UCE, the patient manifested dry symptoms. Normally, clinical manifestations do not occur simultaneously, which was not seen in this patient since she presented with concomitant pictures. Conclusion: The case portrays the typical occurrence of CHU associated with SS and highlights the importance of a good history taking and physical examination. In addition, it draws attention to the association between UCE and other immune-mediated diseases.
\end{abstract}

Keywords: Spontaneous chronic urticaria; Sjögren's Syndrome; Autoimmune. 


\section{Resumen}

Introducción: La Urticaria Crónica Espontánea (UCE) se caracteriza por pápulas transitorias, angioedema o ambos durante más de seis semanas. El UCE puede estar asociado al Síndrome de Sjögren (SS). Objetivo: Se trata de describir un caso clínico de un paciente diagnosticado de Urticaria Crónica Autoinmune, sin mejoría con el tratamiento estándar. Metodología: Este estudio de caso se realizó de forma cualitativa, descriptiva, observacional y retrospectiva, mediante la recogida directa de datos del paciente a través del acceso a los prontuarios y exámenes. Descripción del caso: Paciente femenina, de 34 años, remitida para evaluación reumatológica con UCE. En la búsqueda de causas secundarias para el CHU, presentó exámenes de investigación con anormalidades de laboratorio. Además, también se quejaba de síntomas de sequedad. Los exámenes concluyeron que se trataba de un caso de CHU asociado a SS. Tras el tratamiento de la enfermedad reumática, presentó una mejora de las lesiones urticariales. Discusión: En el caso reportado las lesiones fueron refractarias incluso después de la introducción del tratamiento antihistamínico. Algunas evidencias científicas sugieren mayores tasas de refractariedad al tratamiento estándar del ECU en aquellos pacientes que presentan eventos inmunomediados. Además del cuadro sugestivo de UCE, la paciente manifestaba síntomas de sequedad. Normalmente, las manifestaciones clínicas no ocurren simultáneamente, lo que no se visualizó en la paciente ya que presentó cuadros concomitantes. Conclusión: El caso retrata la ocurrencia típica de CHU asociada a SS y destaca la importancia de una buena historia clínica y examen físico. Además, llama la atención sobre la asociación entre el ECU y otras enfermedades inmunomediadas.

Palabras clave: Urticaria crónica espontánea; Síndrome de Sjogren; Autoinmune.

\section{Introdução}

A Urticária Crônica Espontânea (UCE) é uma doença caracterizada clinicamente pela recorrência de pápulas transitórias, angioedema ou ambos por mais de seis semanas, tendo o prurido como o sintoma mais comumente observado e fisiopatologicamente pela ativação exacerbada de mastócitos e basófilos com liberação de mediadores inflamatórios (Bracken et al., 2019; Hon et al., 2019; Kolkhir et al., 2017). As lesões cutâneas urticariformes se apresentam, geralmente, de forma arredondada ou anular, elevada, bem circunscrita, podendo variar de alguns milímetros a vários centímetros de diâmetro. Além disso, áreas submetidas à pressão e fricção são mais frequentemente acometidas (Hon et al., 2019). Os quadros de UCE ocorrem caracteristicamente na ausência de um fator desencadeante identificável (Bracken et al., 2019). Seu diagnóstico é clínico, realizado através de uma anamnese detalhada e exame físico cuidadoso, e seu tratamento é feito pelo controle sintomático com uso de anti-histamínico e, mais recentemente, as terapias biológicas, como o omalizumabe (anti-IgE) (Ensina et al., 2019; Kolkhir et al., 2017; Saini \& Kaplan 2018).

A Síndrome de Sjögren (SS) é uma doença autoimune que possui como característica marcante sintomas secos, como a xeroftalmia e xerostomia, consequentes à inflamação crônica das glândulas exócrinas por uma infiltração linfoplasmocitária (Parisis D et al., 2020; Zandonella Callegher et al., 2020). Suas manifestações podem ser divididas em glandulares, que levam aos sintomas secos, e extra-glandulares, que correspondem as alterações dos mais variados órgãos e sintomatologias (Muñoz et al., 2004). Além disso, essa doença possui uma maior incidência e prevalência também no sexo feminino. O seu diagnóstico é realizado pela história clínica do paciente, exame físico, avaliação dos sintomas secos oculares e orais específicos, biópsia e exames de imagem das glândulas salivares, além de pesquisa de autoanticorpos (anti-Ro) (Felberg \& Dantas, 2006). Clinicamente, a SS pode ser classificada em primária e secundária, sendo que no primeiro caso a doença ocorre isoladamente, sem a presença de outra doença de tecido conjuntivo. Já na forma secundária é acompanhada de outra doença autoimune (Romão et al., 2018).

Em pacientes com UCE, a associação com outras doenças autoimunes é reconhecida (Bracken et al., 2019). Um dos fatores que leva a suspeição da associação entre UCE e doenças autoimunes é a detecção de anticorpos antinucleares através da imunofluorescência, como a pesquisa do fator antinuclear (FAN) (Magen et al., 2015).

O objetivo do presente estudo foi descrever um caso clínico de uma paciente com diagnóstico recente de Urticária Crônica Espontânea, sem melhora com tratamento padrão e encaminhada para reumatologia para investigação de doenças autoimunes, visto positividade do fator antinuclear (FAN). 


\section{Metodologia}

Este estudo de caso foi realizado qualitativamente, descritiva (Pereira et al., 2018), observacional e retrospectiva, por coleta direta de dados da paciente por meio do acesso aos prontuários e resultados de exames complementares. Os dados foram coletados após o diagnóstico da paciente de Urticária Crônica Espontânea em associação com Síndrome de Sjögren atendida em ambulatório de especialidades pelo pesquisador responsável. Assim, foram analisados dados da história clínica, exames diagnósticos e tratamento da paciente em questão. Este estudo de caso teve aprovação do Comitê de Ética em Pesquisa, com assinatura do Termo de Consentimento Livre e Esclarecido pela paciente.

\section{Descrição do Caso}

Paciente do sexo feminino, 34 anos, encaminhada pelo serviço de alergia e imunologia para avaliação reumatológica de quadro de UCE sem angioedema diagnosticada em maio de 2019. A mesma apresentava lesões urticariformes difusas de aparecimento espontâneo (Figura 1), sem fator desencadeante. Durante investigação de causas secundárias para urticária crônica, foram evidenciados os seguintes achados laboratoriais: imunoglobulina E (IgE) elevada, fator antinúcleo (FAN) padrão nuclear pontilhado fino denso (NPFD) com titulação de 1/640 e fator reumatoide (FR) positivo. A paciente estava em uso de anti-histamínico, porém apresentando refratariedade dos sintomas cutâneos.

Figura 1. Lesões urticariformes em face medial de coxa.

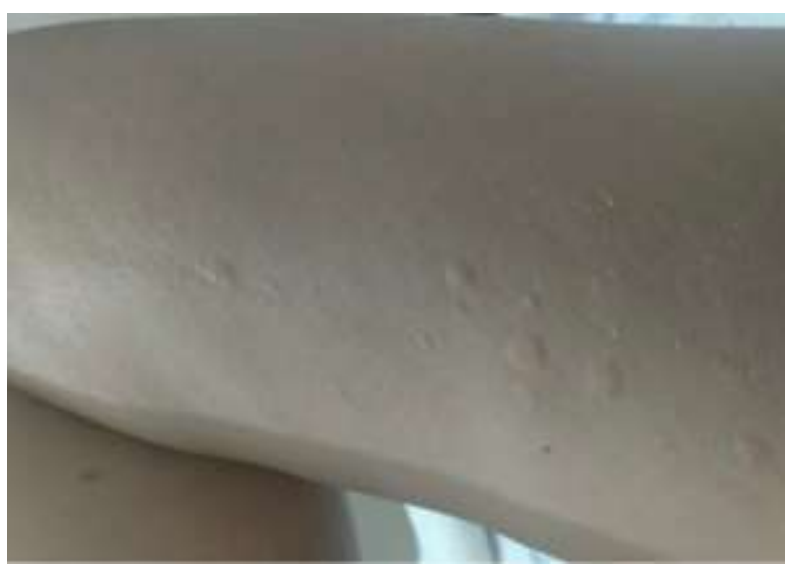

Fonte: Autores.

Durante a avaliação reumatológica, além dos sintomas cutâneos mencionados acima, queixava-se de xerostomia, hiperemia conjuntival bilateral, lacrimejamento excessivo, xerodermia e sintomas prévios sugestivos de parotidite.

Tendo em vista a sintomatologia apresentada e as alterações laboratoriais iniciais mencionadas anteriormente, foram solicitados alguns exames complementares, tais como hemograma, transaminase oxalacética (TGO), transaminase pirúvica (TGP), ureia, creatinina, proteína c-reativa (PCR), lipase sérica, glicemia de jejum, hemoglobina glicada, anti-CCP, anti-La e sorologias para HIV, hepatite B, hepatite C, VDRL e HTLV. Todos esses normais. Foram identificadas sorologias IgG positivas para toxoplasmose, rubéola e citomegalovírus. Além disso, apresentava velocidade de hemossedimentação (VHS) elevada (41 mm na $1^{a}$ hora), amilase sérica de $90 \mathrm{U} / \mathrm{L}$ (VR 22-80 U/L) e anti-Ro $240 \mathrm{U} / \mathrm{mL}$ (VR < $7 \mathrm{U} / \mathrm{mL}$ ). A avaliação oftalmológica objetiva comprovou olho seco e a biópsia de glândula salivar evidenciou sialoadenite crônica não granulomatosa (Figura 2). 
Figura 2. Biópsia de glândulas salivares.

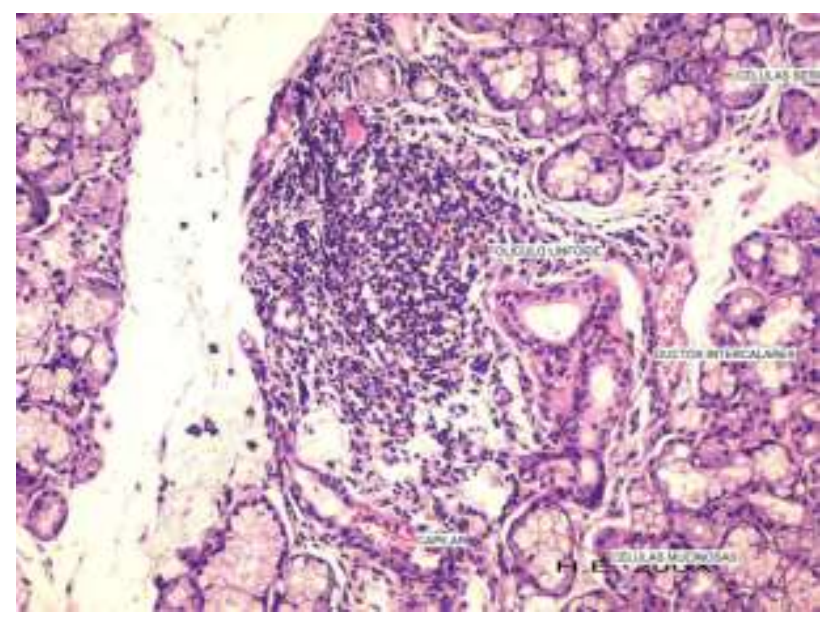

Fonte: Autores.

Diante dos aspectos clínicos, laboratoriais e histopatológicos mencionados anteriormente, concluiu se tratar de um caso de Urticária Crônica Espontânea associada à Síndrome de Sjögren. Nesse momento, foi iniciado tratamento farmacológico com hidroxicloroquina $400 \mathrm{mg} / \mathrm{dia}$, prednisolona $10 \mathrm{mg} /$ dia e colírio lubrificante, além de orientações quanto as medidas para amenizar os sintomas secos.

Em consulta de retorno, após oito meses do diagnóstico, a paciente apresentava melhora dos sintomas glandulares e cutâneos, além disso, negava artralgias e outras queixas. Os exames complementares, incluindo as provas de atividade inflamatória, estavam normais. Foi optado por iniciar desmame da corticoterapia, manutenção do antimalárico, reforço das orientações gerais e acompanhamento clínico ambulatorial.

\section{Discussão}

A UCE é uma doença caracterizada por lesões cutâneas urticariformes, sendo que esses sintomas surgem de forma espontânea, sem identificação de qualquer fator externo desencadeante (Costa \& Gonçalo, 2016). A fisiopatologia não é bem compreendida, mas está claro que o desarranjo da ativação e degranulação de mastócitos e basófilos permanecem como mecanismos centrais na doença (Bracken et al., 2019).

Normalmente, os sintomas se iniciam entre a terceira e quinta década de vida e costumam ser mais incidentes no sexo feminino, numa proporção de duas mulheres para um homem acometido (Hon et al., 2019). No caso, a paciente se enquadra nesse perfil epidemiológico característico da UCE.

A paciente descrita no relato acima, apresentava quadro de urticária crônica sem angioedema, com lesões urticariformes difusas de aparecimento espontâneo. A UCE é caracterizada pela recorrência de pápulas transitórias, angioedema ou ambos com evolução crônica, ou seja, maior que 6 semanas (Kaplan \& Greaves, 2019; Kolkhir et al., 2017). As lesões se apresentam na forma de edema não depressível, bem circunscritas e com centro mais claro e bordas elevadas, envolvendo apenas as porções superficiais da derme sendo vistas em conjunto com o eritema circundante da pele (Bracken et al., 2019).

A maioria dos casos de UCE podem ser diagnosticados clinicamente após uma história detalhada com a duração de pápulas episódicas e transitórias por um período de seis semanas ou mais, uso de medicamentos, estímulos físicos, e exame físico cuidadoso, considerando as pápulas eritematosas edematosas que aumentam e diminuem rapidamente, com ou sem 
angioedema associado. Os diagnósticos diferenciais de vasculite urticariforme, bem como urticária autoimune e autoinflamatória associada, precisam de consideração cuidadosa em investigações clínicas e laboratoriais (Hon et al., 2019).

Uma parte considerável dos pacientes com UCE, cerca de 35\% a 50\% desses, apresentam remissão espontânea dos sintomas de urticária e/ou angioedema em um ano, com duração média da doença de dois a cinco anos (Johal \& Saini, 2020). No caso, não houve remissão espontânea dos sintomas da doença de base. Pelo contrário, as lesões foram refratárias mesmo após introdução do tratamento com anti-histamínico. Algumas evidências científicas sugerem maiores taxas de refratariedade ao tratamento padrão da UCE naqueles pacientes que apresentam associação com eventos imunomediados (Duarte et al., 2018).

Em pacientes com UCE, a associação com outras doenças autoimunes é descrita na literatura médica. Dentre as doenças autoimunes associadas estão a Artrite Reumatoide, Síndrome de Sjögren, Doença Celíaca, Diabetes Mellitus tipo I e Lúpus Eritematoso Sistêmico (Bracken et al., 2019). Além do quadro cutâneo sugestivo de UCE, a paciente apresentava xerostomia, hiperemia conjuntival bilateral, lacrimejamento excessivo, xerodermia e sintomas prévios sugestivos de parotidite, quadro indicativo de Síndrome de Sjögren (SS). O reconhecimento dessa associação com outras doenças imunomediadas é indispensável. A avaliação clínica de outros sintomas, além das lesões urticariformes, é de fundamental importância nesses casos.

A SS é uma doença imunomediada caracterizada por síndrome seca, mais prevalente no sexo feminino, com uma razão de 9 mulheres para 1 homem acometido e início da sintomatologia ocorrendo entre 40 e 60 anos (Parisis D et al., 2020; Rizzo et al., 2020). Além dos sintomas secos, a paciente em questão também apresenta características epidemiológicas compatíveis com SS. Pacientes com UCE apresentam risco relativo 15 vezes maior de desenvolver SS que a população em geral (Confino-Cohen et al., 2012). Naqueles pacientes com UCE e SS associadas, a SS tende a ocorrer cerca de 3 a 5 anos após o início dos sintomas da UCE (Chiu et al., 2018), o que não aconteceu no caso descrito, visto que nesse as manifestações clínicas se apresentaram praticamente de forma simultânea.

Além da sintomatologia extracutânea, outro fator sugestivo da associação entre UCE e doenças imunomediadas é a presença de anticorpos antinucleares identificados através do FAN (Magen et al., 2015). Esse achado associado à refratariedade ao tratamento anti-histamínico foram fundamentais para investigação reumatológica adicional nesse caso.

Em relação ao tratamento, a hidroxicloroquina (HCQ) tem sido comumente usada há muitos anos para controle de doenças reumáticas imunomediadas, dentre elas a SS, devido sua ação imunomodulatória capaz de reduzir as vias inflamatórias (Danza et al., 2016). No caso, a paciente acima apresentou boa resposta ao tratamento imunomodulador instituído, sendo possível realizar desmame da corticoterapia.

Geralmente, casos de UCE associada à doença imunomediada apresentam maior taxa de remissão do quadro cutâneo comparados a UCE isolada. Em contrapartida, as doenças imunomediadas podem impactar negativamente a qualidade de vida do paciente, exigindo tratamentos adicionais voltados para controle dos sintomas das mesmas (Confino-Cohen et al., 2012).

\section{Conclusão}

Trata-se de um caso com diagnóstico praticamente simultâneo de UCE e SS, o que o difere da literatura. Pacientes com UCE têm risco aumentado de SS, logo, esta doença imunomediada deve fazer parte do grupo de doenças investigadas nos casos de UCE, principalmente naqueles com refratariedade ao uso de anti-histamínicos, na presença de sintomas secos e FAN positivo. A associação entre UCE com outras doenças imunomediadas pode impactar negativamente no prognóstico das mesmas. Portanto, é de extrema importância a realização de uma anamnese e exame físico detalhados com finalididade de identificar possíveis sintomas extracutâneos sugestivos de outras doenças imunomediadas. 
Além disso, dados clínicos podem ser úteis na suspeita da associação entre UCE e doenças imunomediadas, tais como a presença de outros sintomas além das lesões urticariformes e angioedema, presença do FAN e refratariedade ao tratamento padrão para UCE. Apesar de descrita na literatura, essa associação entre UCE e doenças imunomediadas ainda é pouco divulgada na prática clínica. Sendo assim, faz-se necessário mais pesquisas sobre relatos de casos clínicos ou série de casos, que devem ser mais abrangentes a fim de ajudar os profissionais de saúde no diagnóstico precoce da UCE e sua associação com a SS ou outras doenças imunomediadas. Dessa forma, poderá refletir também em trabalhos futuros, uma vez que o diagnóstico mais precoce dessa associação pode ter impacto positivo na evolução do quadro clínico.

\section{Referências}

Bracken, S. J, Abraham, S., \& MacLeod, A. S. (2019). Teorias autoimunes da urticária espontânea crônica. Frontiers in immunology, $10,627$.

Chiu, H. Y., Muo, C. H., \& Sung, F C. (2018). Associações de urticária crônica com comorbidades atópicas e autoimunes: um estudo nacional de base populacional. International Journal of Dermatology, 57 (7), 822-829.

Confino-Cohen, R., Chodick, G., Shalev, V., Leshno, M., Kimhi, O., \& Goldberg, A. (2012). Urticária crônica e autoimunidade: associações encontradas em um grande estudo populacional. Journal of alergia e imunologia clínica, 129 (5), 1307-1313.

Costa, C., \& Gonçalo, M. (2016). On behalf of GPEU-Grupo Português de Estudos de Urticária. Diagnostic and therapeutic approach of chronic spontaneous urticaria: recommendations in Portugal. Acta Med Port, 29, 763-81.

Danza, Á., Graña, D., Goñi, M., Vargas, A., \& Ruiz-Irastorza, G. (2016). Hidroxicloroquina en el tratamiento de las enfermedades autoinmunes sistémicas. Revista médica de Chile, 144 (2), 232-240.

Duarte, R. A. G., de Castro, R. B., Almonfrey, F. B., Kalil, J., Motta, A. A., \& Agondi, R. C. (2018). Características clínicas e autoimunidade nos pacientes com urticária dermográfica. Arquivos de Asma, Alergia e Imunologia, 2(4), 434-440.

Ensina, L. F., Sano, F., Silva, E. K., Rubini, N. D. P. M., \& Solé, D. (2019). Omalizumabe no tratamento da urticária crônica espontânea: análise de custoefetividade e impacto orçamentário. Arquivos de Asma, Alergia e Imunologia, 3(1), 51-63.

Felberg, S., \& Dantas, P. E. C. (2006). Diagnóstico e tratamento da síndrome de Sjögren. Arquivos brasileiros de oftalmologia, 69(6), 959-963.

Hon, K. L, Leung, A. K, Ng, W. G., \& Loo, S. K. (2019). Urticária crônica: uma visão geral do tratamento e patentes recentes. Patentes recentes sobre descoberta de medicamentos para inflamação e alergia, 13 (1), 27-37. https://repositorio.ufsm.br/bitstream/handle/1/15824/Lic_Computacao_MetodologiaPesquisa-Cientifica.pdf?sequence $=1$.

Johal, K. J., \& Saini, S. S. (2020). Current and emerging treatments for chronic spontaneous urticaria. Annals of allergy, asthma \& immunology: official publication of the American College of Allergy, Asthma, \& Immunology, 125(4), 380-387. https://doi.org/10.1016/j.anai.2019.08.465

Kaplan, A. P, \& Greaves, M. (2009). Patogênese da urticária crônica. Clinical \& Experimental Allergy, 39 (6), $777-787$.

Kolkhir, P., Church, M K, Weller, K., Metz, M., Schmetzer, O., \& Maurer, M. (2017). Urticária espontânea crônica autoimune: o que sabemos e o que não sabemos. Journal of Allergy and Clinical Immunology, 139 (6), 1772-1781.

Magen, E., Waitman, D. A, Dickstein, Y., Davidovich, V., \& Kahan, N. R. (2015). Características clínico-laboratoriais da urticária idiopática crônica ANApositiva. Em Allergy \& Asthma Proceedings (Vol. 36, No. 2).

Muñoz, M. D. G. H. D. C. N., Castelão, W. C. B., Saraiva, F. M. D., Costa, J. C. T. D., \& Queiroz, M. F. O. V. D. (2004). Síndrome de Sjögren primária: manifestações exócrinas e não exócrinas. Revista Brasileira de Reumatologia, 44, 129-138.

Parisis D, Chivasso C, Perret J, Soyfoo M. S, \& Delporte C. Current State of Knowledge on Primary Sjögren's Syndrome, an Autoimmune Exocrinopathy. Journal of Clinical Medicine. 2020;9(7).

Pereira, A. S. et al. (2018). Metodologia da pesquisa científica. UFSM.

Rizzo, C., Grasso, G., Destro Castaniti, G. M., Ciccia, F., \& Guggino, G. (2020). Síndrome de Sjogren primária: enfoque nas células imunes inatas e na inflamação. Vaccines, 8 (2), 272.

Romão, V. C, Talarico, R., Scirè, C. A, Vieira, A., Alexander, T., Baldini, C., \& Mariette, X. (2018). Síndrome de Sjögren: estado da arte em diretrizes de prática clínica. RMD aberto, 4 (Suplemento 1), e000789.

Saini, S. S., \& Kaplan, A P. (2018). Urticária espontânea crônica: a coceira do diabo. The Journal of Allergy and Clinical Immunology: In Practice, 6 (4), 1097-1106.

Zandonella Callegher, S., Zabotti, A., Giovannini, I., Treppo, E., Quartuccio, L., \& De Vita, S. (2020). A ultrassonografia de glândula salivar de aparência normal identifica um fenótipo mais moderado da síndrome de Sjögren primária. Fronteiras na medicina, 7, 910. 